\title{
Neoclassical Tearing Modes in Tokamak Fusion Test Reactor Experiments Part I. Measurements of Magnetic Islands and $\Delta^{\prime}$
}

\author{
Z. Chang, E. D. Fredrickson, S. H. Batha ${ }^{1}$, M. G. Bell, R. V. Budny, F. M. Levinton ${ }^{1}$, \\ K. M. McGuire, G. Taylor, M. C. Zarnstorff and TFTR group \\ Plasma Physics Laboratory, Princeton University, Princeton, NJ 08543 \\ ${ }^{1}$ Fusion Physics and Technology, Torrance, CA
}

(November 3, 1997)

\begin{abstract}
Tearing type modes are observed in most high confinement operation regimes in TFTR. Three different methods are used to measure the magnetic island widths: external magnetic coils, internal temperature fluctuation from electron cyclotron emission (ECE) diagnostic and an experiment where the plasma major radius is rapidly shifted ("Jog" experiments). A good agreement between the three methods is observed. Numerical and analytic calculations of $\Delta^{\prime}$ (the tearing instability index) are compared with an experimental measurement of $\Delta^{\prime}$ using the tearing mode eigenfunction mapped from the jog data. The obtained negative $\Delta^{\prime}$ indicates that the observed tearing modes cannot be explained by the classical current-gradient-driven tearing theory.
\end{abstract}

(To be submitted to Physics of Plasmas for publication.) 


\section{INTRODUCTION}

Tearing modes and magnetic islands $[1,2]$ are fundamental physical phenomena in magnetized plasmas. The existence of large magnetic islands can degrade or even terminate the performance of tokamak plasmas.[3] In small and medium size tokamaks without auxiliary heating, where plasma temperature is relatively low, tearing modes are believed to be mainly driven by the current gradient $(\nabla J, J$ is the inductive current) and the magnetic islands are believed to basically follow the Rutherford nonlinear evolution equation [4]. We call these modes classical tearing modes. When auxiliary heating is introduced, e.g., neutral beam injection (NBI) and various radio-frequency $(\mathrm{RF})$ heating schemes, plasma temperature increases and the plasmas becomes more collisionless. When the ion-ion collision frequency $\left(\nu_{i}\right)$ becomes smaller than the ion banana bounce frequency $\left(\omega_{b}\right)$, the existence of magnetically trapped particles and their interaction with non-trapped (circulating) particles will generate a bootstrap current [5] in the region of large plasma pressure gradient $(\nabla P)$. Theoretically, this non-inductive current or $\nabla P$ term can also drive tearing modes and produce magnetic islands $[6,7]$, especially when $\Delta^{\prime}<0$ and the classical $\nabla J$-term no longer plays a driving role, where $\Delta^{\prime}$ is the well-known tearing instability index. This new class of tearing instability, the so-called neoclassical tearing mode, was first identified in TFTR[8]. Neoclassical tearing modes have also been observed in other tokamaks, $[9,10]$. Recently, the observation of tearing modes in plasmas with reversed magnetic shear has provided experimental evidence supporting the neoclassical tearing theory. Meanwhile, new theories have been recently developed to include more physical effects and introducing models for the threshold island width. These new models provide more tools to study the complex experimental observations.

Detailed study of the nonlinear tearing instability and island evolution requires accurate measurements of magnetic islands and experimental justification of the $\Delta^{\prime}$. These two topics are addressed in this paper. Section II shows three 
ways of measuring island width: external magnetic measurement, internal local temperature measurement and a direct measurement from a major radius shift experiment (plasma "Jog"). A general agreement between these different methods is observed. Section III discusses the $\Delta^{\prime}$ evaluation using numerical calculations and analytic formulas. The results are compared with the direct experimental measurement of $\Delta^{\prime}$ using the data from the Jog experiment. The conclusion of part I of this paper is given in Section IV. In a companion paper to be published subsequently, we will show that the neoclassical tearing theory provides a very promising model to explain many experimental observations.

\section{MEASUREMENT OF MAGNETIC ISLANDS}

\section{A. MHD diagnostics in TFTR}

Many diagnostics can be used to detect MHD activity (hereafter referred to simply as MHD) in TFTR. We will present results from two main diagnostics: magnetic coils (Mirnov coils) [11] and electron cyclotron emission (ECE) grating polychromator (GPC) system[12]. These are illustrated in Fig. 1. There are 30 Mirnov coils spread around the torus. The coils are mounted between the vacuum vessel and the bumper limiter (high field side) or RF limiter (low field side). The distance between the vacuum vessel and coils is about $10 \mathrm{~cm}$ (so the Mirnov coils are on a circle with minor radius of $110 \mathrm{~cm}$ ). There are two independent 20 channel ECE (second harmonic) GPC arrays. They are toroidally separated by $126^{\circ}$. These two arrays measure electron temperature and its fluctuation profiles along the midplane. The channel-to-channel separation is typically $5-6 \mathrm{~cm}$. Each channel has a spatial resolution about $2-3 \mathrm{~cm}$ radially and $\sim 10 \mathrm{~cm}$ vertically. To avoid overlap with the $3^{\text {rd }}$ harmonic emission, the grating angles are usually set so that the inner most channel is at a major radius of $220-230 \mathrm{~cm}$. Since the GPCs are not absolutely calibrated, another ECE diagnostic system, a Michelson interferometer, [13] is routinely used for calibration. 
Figure 2 shows a typical neutral-beam heated, high-performance discharge (supershot [14]) that developed a $m / n=3 / 2$ mode, where $m$ is the poloidal mode number and $n$ is the toroidal mode number. Significant degradation in the Troyon-normalized beta $\left[\beta_{N}=\beta_{t}(\%) a(m) B_{t}(T) / I_{p}(M A)\right]$ and neutron rate are observed, which are correlated with the MHD. The Fourier spectrum evolution from one Mirnov coil is shown in Fig. 2(b). The $3 / 2$ mode starts at about $3.8 \mathrm{~s}$ (300 ms after NBI) preceded by an $m / n=1 / 1$ fishbone-like mode. There is a $\sim 150$ ms overlap period where both modes co-exist. The $3 / 2$ mode decays after the NB phase and is terminated by the injection of Lithium pellet at $5.03 \mathrm{~s}$.

The measured $T_{e}$ profile is shown in Fig. $3(\mathrm{a})$. A $18 \mathrm{kHz}$ mode is clearly seen on some of the internal ECE channels, see Fig. 3(b). The fluctuating component

of the electron temperature $\tilde{T}_{\epsilon}$ phase reversal is seen at about $R=305 \mathrm{~cm}$ $(r / a \simeq 0.41)$, indicating the mode has a tearing parity. The odd phase of the mode on both sides of the magnetic axis ( Ch 1 and $\mathrm{Ch} 11$ ) confirms that the poloidal mode number $m$ is 3 . Also, using the two toroidally separated ECE systems, we obtained $n=2$ in agreement with the magnetic measurement.

\section{B. Island width from magnetic measurement}

Magnetic island widths can be calculated using external Mirnov coil measurement. We use the following well-known cylindrical formula:

$$
W=4 r_{s} \sqrt{\frac{1}{m} \frac{\tilde{B}_{r}}{B_{\theta}} \frac{q}{\left|q^{\prime}\right| r}} \quad \text { at } r=r_{s}
$$

Here, $B_{\theta}$ is the equilibrium poloidal field, $q^{\prime}=d q / d r, r_{s}$ denotes the radial location of the mode. The local radial magnetic perturbation $\tilde{B}_{r}$ is calculated numerically by solving the MHD equation [16]:

$$
r \frac{d}{d r} r \frac{d \tilde{\psi}}{d r}-m^{2} \tilde{\psi}-\frac{q}{1-n q / m} r \frac{d}{d r}\left(\frac{1}{r} \frac{d}{d r} \frac{r^{2}}{q}\right) \tilde{\psi}=0 .
$$

Here, $\tilde{\psi}$ is the perturbed poloidal magnetic flux, related to the magnetic perturbation through $\tilde{\mathbf{B}}=\nabla \tilde{\psi} \times \hat{\mathbf{z}}, \tilde{B}_{\theta}=-\partial \tilde{\psi} / \partial r, \tilde{B}_{r}=i m \tilde{\psi} / r$. The fixed-boundary 
conditions are used for simplicity, i.e., $\tilde{B}_{r}($ coil $)=0, \tilde{B}_{\theta}($ coil $)=\tilde{B}_{p c}$, where coil denotes the coil location and $\tilde{B}_{p c}$ is the measured mode amplitude. (We use the data from the coil at the $60^{\circ}$ below the midplane indicated in Fig. 1, which usually has an average amplitude among the poloidal array.)

Knowing the correct $q$ profile is crucial for the island width calculation. The $q$ profile measured by the Motional Stark Effect (MSE) diagnostic[18] is used when it is available. However, due to conflicting requirements for the neutral beam configuration, most high power supershot discharges do not have reliable MSE measurements. We will use $q$ profiles from self-consistent transport calculations using the TRANSP code [15]. Since the TRANSP $q$ does not always agree with the MHD measurement when the mode location can be identified, a mapping of the TRANSP $q(r)$ is used in order to match the measured mode rational surface location $\left(r_{s}\right)$ :

$$
\hat{q}(r)=q(r)\left[1+\left(\frac{m}{n q\left(r_{s}\right)}-1\right) \frac{(1-r / a)}{\left(1-r_{s} / a\right)}\right],
$$

where $\hat{q}$ is the modified safety factor. Note that this modification does not change $q_{a}$. For example, for the case shown in Fig. 3, the calculated island width is about $7.7 \mathrm{~cm}(W / a=8.9 \%)$ using the modified $q$, while the original $q$ gives $W \simeq 6.2 \mathrm{~cm}$. Justification of this larger width result is given in the following sub-section.

\section{Island width from local $\tilde{T}_{e}$ measurement}

The easiest way to obtain the island width of an MHD mode would be to measure the "flat spot" in electron temperature profile. However, in TFTR the large ECE channel spacing $(5-6 \mathrm{~cm})$ and uncertainty in the channel to channel calibration make this method unreliable except for the case of a very large island. However, after some investigation we found that the $T_{e}$ fluctuation data from one or two channels around the mode rational surface could be used to estimate the island width. First, we consider the case where one ECE channel is located inside the island and therefore shows a deformed wave form as shown in Fig. 4. 
Based on a slab model for the island geometry and a constant- $\psi$ approximation, we found that the island width is given by (see Appendix A)

$$
W=\frac{\delta T}{\left|T^{\prime}\right|} \frac{2 \sqrt{2}}{\sqrt{1-\cos \zeta_{c}}} .
$$

Here, $\delta T$ is the residual fluctuation amplitude (shown as channel $b$ in Fig. 4). The phase angle $\zeta_{c}$, which is the phase when the channel starts to enter the island, is equal to $2 \pi f t_{c}$, where $f$ is the mode frequency.

When an island is located between two channels, so that the width is smaller than the distance between the two channels, we can estimate the island width using the relation (valid for the outer region, $\left|r-r_{s}\right|<W / 2$ ):

$$
\tilde{T}_{e}=-\nabla T_{e} \cdot \xi=-T_{e}^{\prime} \frac{\tilde{\psi}}{B_{\theta}\left(1-q / q_{s}\right)} .
$$

Here, $\xi$ is the plasma displacement due to the mode. A simple estimation of the island width can be obtained (see Appendix A):

$$
W \simeq \sqrt{2 d \frac{\tilde{T}}{\left|T^{\prime}\right|}} \quad \text { for } W \lesssim d
$$

where $d$ is the distance between two channels and $\tilde{T}$ is the averaged fluctuation level (peak-to-peak) of the two channels for the case where the island is not at the middle. In practice, we use Fourier analysis to select the tearing mode and use TRANSP equilibrium mapping to obtain $T^{\prime}\left(r_{s}\right)$.

Figure $5\left(\right.$ a) shows the $\tilde{T}_{e}$ data at different phases of the $3 / 2$ island evolution in the discharge of Fig. 2. The island evolution from Mirnov coil data (using the corrected $q$ profile discussed in previous sub-section) is shown in Fig. 5(b). Also shown for comparison is the measured island width using ECE data and Eq. (6). For the first two periods during which the ECE data was recorded at high rate $(500 \mathrm{kHz})$ around $3.85 \mathrm{~s}$ and $4.0 \mathrm{~s}$ the $\tilde{T}_{e}$ is the average of channels 12 and $13 . \mathrm{In}$ the time windows around $4.2 \mathrm{~s}, 4.4 \mathrm{~s}$ and $4.75 \mathrm{~s}$ channel 12 is partly crossing the island so we used channels 13 and 11 to obtain the averaged $\tilde{T}_{e}$. At the last time 
window around $4.9 \mathrm{~s}$, the mode moves inward, so we used channels 12 and 10 to get the averaged $\tilde{T}_{e}$. We have also used the data from the channel that crosses the island so that Eq. (4) can be used. For example, using channel 12 at $4.75 \mathrm{~s}$,

the deformed $\tilde{T}_{e}$ wave form gives $W \simeq 7.0 \pm 0.5 \mathrm{~cm}$, which agrees with the outside island measurement. As we can see in Fig. 5 the two independent measurements (Mirnov coil and ECE) are in good agreement. (Note that the corrected $q$ profile is applied in the magnetic calculation as mentioned in Section II.B.)

Similar agreement has also been found for islands with other helicities. Figure 6 shows the island evolution of $m / n=4 / 3$ and $5 / 4$ modes. We found that Eq. (6) provides a very useful estimation of island width when islands are small [but still defined by Eq. (1)]. Using a specially designed direct measurement of island width discussed in the next section, we found that the above indirect measurements of magnetic island width can be justified.

\section{Island width from "Jog" experiments}

If a plasma is shifted or "jogged" radially, each ECE channel will continuously sweep through a portion of the plasma. We call this a plasma "Jog" experiment[17]. If the shift is adiabatic and the magnetic islands are not perturbed, the time trace of the fast ECE measurement can be mapped into $T_{e}(R)$ and $\tilde{T}_{\epsilon}(R)$ profiles with very fine spatial resolution.

Figure 7(a) shows a Jog experiment during the NBI phase. The plasma is compressed and decompressed about $10 \mathrm{~cm}$ during $100 \mathrm{~ms}$. There is a $2 / 1$ mode in this discharge which developed before the Jog. The mapped $T_{e}$ and $\tilde{T}_{e} / T_{e}$ profiles from the jog data are shown in Fig. 7(b). From the magnetic island geometry we know that the distance between the two $\tilde{T}_{e} / T_{e}$ peaks around the mode rational surface is equal to the island width. In this case the $2 / 1$ island is about $7 \mathrm{~cm}$ at the high-field-side. We can also use the local temperature fluctuation data to calculate the island width. For example, during the jog phase 
the $\tilde{T}_{e}(t)$ wave form of three adjacent channels at the high-field-side is shown in Fig. 7(c). Using Ch\#3 for Eq. (4) we obtain $W \simeq 6.8 \mathrm{~cm}$. Using Ch\#2 and Ch\#4 for Eq. (6) we obtain $W \simeq 7.1 \mathrm{~cm}$. Both of these results are in good agreement with the direct Jog measurement of $W \simeq 7.0 \mathrm{~cm}$. Using the MSE $q$ profile we can calculate the island width from the Mirnov data: $W \simeq 6.5 \pm 1.0$ cm. Similar good agreement between all three methods has also been observed for $3 / 2$ island cases.

The agreement between the measured island width and magnetically calculated width has two important implications. First of all, since the plasma Jog is only used in rare test cases, we can confidently use the magnetic and ECE data to calculate the island width and its time evolution. Secondly, since the $q$ profile is important in calculating the island width from Mirnov data, this agreement implies that the $q$ profile and its first derivative near the island are reasonably correct [see Eq. (1)]. This information can be used to support the calculation of the $\Delta^{\prime}$ parameter discussed in the next Section.

\section{EVALUATION OF $\Delta^{\prime}$}

The well-known tearing instability index $\Delta^{\prime}$ is defined by

$$
\Delta^{\prime}=\left.\frac{d \tilde{\psi} / d r}{\tilde{\psi}}\right|_{r s+}-\left.\frac{d \tilde{\psi} / d r}{\tilde{\psi}}\right|_{r s-} .
$$

Theoretically, it is a robust parameter that determines the classical tearing instability, i.e., the these modes should be unstable for $\Delta^{\prime}>0$ and stable otherwise. However, it is not a directly measurable physical quantity. We present here three different approaches of the $\Delta^{\prime}$ evaluation: (1) Numerical integration of Eq. (2). This is a well-known and the most commonly used approach. (2) Analytic formula for large $m$ modes. (3) Direct evaluation using the ECE measured $\delta T_{e} / T_{e}$ profile in the Jog experiment. The first two approaches require knowledge of $q$ profile and its first and second derivatives around the mode rational surface [see Eq. (8) below]. We will first discuss the second approach and compare the results 
with the first numerical approach.

\section{A. Analytic formula and numerical calculation}

For large $m$ and cylindrical geometry, an analytic formula was derived by Strauss[19] and later generalized by Hegna and Callen[20]:

$$
\Delta^{\prime}=-\frac{2 m}{r_{s}} \frac{\pi \sigma / 2}{\tan (\pi \sigma / 2)}
$$

where

$$
\begin{aligned}
\sigma & \equiv \frac{r_{s}}{m} \frac{q}{B_{\theta}} \frac{\mu_{0}\left|d J_{||} / d r\right|}{d q / d r}=-\frac{1}{m} \frac{q^{2}}{d q / d r} \frac{d}{d r}\left[\frac{1}{r} \frac{d}{d r}\left(\frac{r^{2}}{q}\right)\right] \\
& =\frac{1}{m}\left(3+\frac{r q^{\prime \prime}}{q^{\prime}}-\frac{2 r q^{\prime}}{q}\right) .
\end{aligned}
$$

For $m \gg 1 \mathrm{Eq}$. (8) leads to the well-known large $m$ result, $\Delta^{\prime} \sim-2 m / r_{s}$. The comparison of the $\Delta_{c y l}^{\prime}$ between the numerical integration of Eq. (2) and Eq. (8) is shown in Fig. 8(a). The TRANSP $q$ profile is shown in Fig. 8(b). It can be partly justified by the locations of the observed MHD, namely, fishbone-type $m / n=1 / 1$ mode and $m / n=4 / 3$ tearing mode. A polynomial fit to the TRANSP data was used in the calculation. As we can see, Eq. (8) agrees well with the numerical results for $m \geq 3$. Note that the large- $m$ limit $\left(-2 m / r_{s}\right)$ is not a good approx-

imation for $m<6$. Since $\Delta^{\prime}<0$ for all the $m \geq 3$ modes, the classical tearing theory would predict that these modes are tearing stable, which is inconsistent with the observation of the $4 / 3$ tearing mode in this discharge. Note that the toroidal/finite- $\beta$ effects[21] [often referred as Glasser-Green-Johnson (GGJ) effects] will make the tearing mode more stable. Therefore, the cylindrical $\Delta^{\prime}$ can be considered an upper limit.

\section{B. Finite island width effect on $\Delta^{\prime}$}

Strictly speaking, the $\Delta_{c y l}^{\prime}$ is also a function of island width. It can be nu- 
merically treated using following definition:

$$
\Delta^{\prime}(W)=\left.\frac{d \tilde{\psi} / d r}{\tilde{\psi}}\right|_{r s+W / 2}-\left.\frac{d \tilde{\psi} / d r}{\tilde{\psi}}\right|_{r s-W / 2}
$$

Namely, we can integrate Eq. (2) to $r_{s} \pm W / 2$ to obtain the $\Delta^{\prime}(W)$. Three examples are shown in Fig. 9 for $m / n=2 / 1,3 / 2$ and $4 / 3$ modes. All the modes are observed in different discharges. The $q$ profiles used are before the MHD phase. It is seen that for $3 / 2$ and $4 / 3$ modes where $\Delta^{\prime}$ s are negative, the $W$ dependence in $\Delta^{\prime}$ is small. For the $2 / 1$ mode, the positive $\Delta^{\prime}$ decays quickly to zero and becomes negative as $W$ increases.

An analytic expression for cylindrical $\Delta^{\prime}(W)$ has also been obtained by Hegna and Callen for high $m$ mode [20]. It is formulated in terms of Whittaker functions, see Eq. (23) in Ref.[20], which can be simplified to the following integral formula:

$$
r_{s} \Delta^{\prime}(W)=-m e^{-\Omega / 2} \int_{0}^{\infty} d t e^{-t}\left[\frac{(t+\Omega+2 \lambda) t^{\lambda}}{(t+\Omega)^{1+\lambda}}+\frac{(t+\Omega-2 \lambda) t^{-\lambda}}{(t+\Omega)^{1-\lambda}}\right] .
$$

Here, the island width dependence is in the parameter $\Omega=m W / r_{s}$. The $q$ profile dependence is included in parameter $\lambda=\sigma / 2$, where $\sigma$ is defined in Eq. (10). The results are shown in Fig. 9. For the $3 / 2$ and $4 / 3$ modes, the $\lambda_{\mathrm{s}}$ are calculated using the TRANSP $q(r)$. As we can see the analytic formula agrees well with the numerical result for $m \leq 3$. For the $2 / 1$ mode, there is usually a significant difference between the numerical and analytic results. To compare the $W$ dependence we normalized the $\Delta^{\prime}(W)$ at the zero island width.

\section{Experimental measurement of $\Delta^{\prime}$}

A possible direct experimental evaluation of $\Delta^{\prime}$ can be obtained based on Eq. (5) by using the ECE data from the Jog experiment. Taking the derivative of Eq. (5) we have

$$
\frac{1}{\tilde{\psi}} \frac{d \tilde{\psi}}{d r}=\frac{1}{B_{\theta}} \frac{d B_{\theta}}{d r}+\frac{1}{L_{T e}} \frac{d L_{T e}}{d r}-\frac{1}{\left(q_{s}-q\right)} \frac{d q}{d r}+\frac{T_{e}}{\tilde{T}_{e}} \frac{d}{d r}\left(\frac{\tilde{T}_{e}}{T_{e}}\right)
$$


Here, $L_{T e}=-d \ln T_{e} / d r$ is the equilibrium temperature scale length. The $\Delta^{\prime}(W)$ can therefore be written as

$$
\Delta^{\prime}(W)=\left\langle\frac{1}{B_{\theta}} \frac{d B_{\theta}}{d r}\right\rangle+\left\langle\frac{1}{L_{T e}} \frac{d L_{T e}}{d r}\right\rangle-\left\langle\frac{1}{\left(q_{s}-q\right)} \frac{d q}{d r}\right\rangle+\left\langle\frac{T_{e}}{\tilde{T}_{e}} \frac{d}{d r}\left(\frac{\tilde{T}_{e}}{T_{e}}\right)\right\rangle,
$$

where $\left.\langle f\rangle \equiv f\right|_{r s+W / 2}-\left.f\right|_{r s-W / 2}$. All the four terms can be measured experimentally, i.e., the equilibrium $B_{\theta}$ and $q$ from time averaged MSE (TRANSP) data, $T_{e}$ and $\tilde{T}_{e}$ from ECE Jog data shown before. Since Eq. (5) is valid only for exterior region, i.e., $\left|r-r_{s}\right|>W / 2$, we use an analytic expression to fit the $\tilde{T}_{\epsilon}$. One example for the $3 / 2$ mode is shown in Fig. 10. Here, the $R \rightarrow r$ mapping of the Jog fluctuation data has been made using TRANSP calculated Shafranov shift. The analytic fitting to the exterior eigenfunction is shown as the solid curves. The four terms of the right side of Eq. (14) as a function of $x=\left(r-r_{s}\right) / a$ are shown in Fig. 11(a). The main contribution is from the competition between the

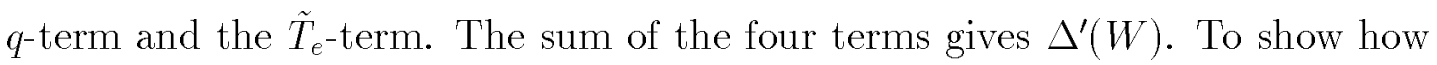
the sum varies with $\left(r-r_{s}\right)$ we plot $r_{s} \Delta^{\prime}$ against $2\left(r-r_{s}\right)$ in Fig. 11(b). Since the $\Delta^{\prime}$ is defined at $2\left(r-r_{s}\right)=W$, which is between 5 and $5.5 \mathrm{~cm}$, we have the ECE measured $r_{s} \Delta^{\prime}(W) \simeq-5.0$. This is in reasonable agreement with the calculated $r_{s} \Delta^{\prime}(W) \simeq-3.5$ using the $q$ profile just before the jog.

The same process has been carried out for the $4 / 3$ island. The results are shown in Fig. 12. A good fit to the jog data yields a negative $\Delta^{\prime}$ which is close to the numerical calculation. To obtain a positive $\Delta^{\prime}$, say $r_{s} \Delta^{\prime} \simeq+5$, the fitting curves would become obviously inconsistent with the jog data, shown as the bad fit in Fig. 12(a). This exercise shows that the negative $\Delta^{\prime}$ from the numerical calculation is confirmed by the jog data when a good fit to the data is used. At last a $2 / 1$ island case is shown in Fig. 13. It is found that the $\Delta^{\prime}(W)$ from the jog data is more negative than the numerical value. Also shown in the figure is the numerical value of $\Delta^{\prime}$ without finite width correction (see Fig. 9). It is worth commenting that the uncertainty of this method is likely to be large. First of all, the $\Delta^{\prime}$ value is deduced from a difference of two big numbers, see Fig. 11(a). 
Second, since each ECE channel has 2-3 cm instrumental spatial resolution, this will effectively smooth the real data. However, the absence of smoothing would make the actual eigenfunction more peaked near the separatrix. This would make the $\Delta^{\prime}$ more negative. The accuracy of $q$ profiles will also affect the results. However, since only the equilibrium value is required in Eq. (14) the lack of spatial resolution may not invalidate this method.

A different method to evaluating $\Delta^{\prime}$ using the Jog ECE data has recently be developed [22]. It is based on a higher order analysis of the magnetic island geometry. Similar results have been achieved for the jog discharges discussed above.

Physically, the negative value of $\Delta^{\prime}$ discussed above indicates that these tearing modes cannot be explained by the classical tearing theory. In Part II of this paper we will show that the negative $\Delta^{\prime}$ is actually required for the saturation of a neoclassical magnetic island. It is found that the neoclassical tearing theory can provide a good explanation to the observations.

\section{CONCLUSIONS}

Using high quality MHD diagnostic data in TFTR, we are able to measure the magnetic island width and estimate the $\Delta^{\prime}$ - two basic physical concepts in plasma physics. Although one can use the external magnetic data to easily calculate the island width, lack of a good $q$ profile, the poloidal asymmetry of the magnetic data and sometimes the noise of measurement contributed by MHD activity at the edge often make the calculation ambiguous and inaccurate. We have shown in this paper that an independent and more direct measurement of island width can be obtained using internal $T_{e}$ fluctuation measurements around the mode rational surface. The formulas developed in this paper [Eq. (4) and Eq. (6)] provide an internal local measurement of island width even when the island width is much smaller than the ECE channel spacing. Since the $q$ profile 
is not required in the calculation, this method is more reliable than the usual magnetic calculation. For large island cases, results from both magnetic and local-ECE measurements are confirmed by the plasma jog experiment where the island width can be directly measured. In addition, the agreement between ECE measurement and magnetic calculation provides an experimental justification for the $q$ profile, which for many cases is not a directly measured quantity.

Numerical and analytic calculation of $\Delta^{\prime}$ have been compared. It is shown that for $m \geq 3$ the analytic formula of Strauss and Hegna-Callen agrees with the numerical results. This agreement also extends to the finite island width effect, i.e., $\Delta^{\prime}(W)$. A new way of estimating the $\Delta^{\prime}(W)$ using the Jog ECE fluctuation data has been discussed. It is found that for the discharges studied (with $m / n=3 / 2,4 / 3$ and $2 / 1$ islands) the $\Delta^{\prime}(W)$ 's are negative. The values are in good agreement (except for the $2 / 1$ island case) with the numerical calculations. These results have important physical meanings. They indicate that the observed tearing modes are not the classical tearing modes driven by current gradient. In Part II we will find that neoclassical tearing theory provides a promising model for explaining many of the experimental observations.

\section{Acknowledgment}

The authors would like to acknowledge the support from R. Hawryluk and valuable discussions with J. Callen, C. Hegna, J. Manickam, D. Monticello, W. Park, and C. Ren. This research is sponsored by the U. S. Department of Energy under Contract No. DE-AC02-76CH03073. 


\section{APPENDIX A. Island width from ECE fluctuation measurements}

In slab geometry, which is a reasonable approximation for small islands in tokamak, the total helical flux in an equilibrium containing a magnetic island can be written as

$$
\psi=-B_{\theta} \frac{q_{s}^{\prime}}{q_{s}} \frac{x^{2}}{2}-\tilde{\psi} \cos \zeta
$$

where $x=r-r_{s}, \tilde{\psi}$ is the perturbation, and $\zeta=(m \theta-n \phi)$ is the helical phase

angle. Since $\tilde{\psi}$ is nearly constant around the island (constant $\psi$ approximation), by following the separatrix flux $\left(\psi_{x}\right)$ at $\zeta=0$ and $\pi$, see Fig. 4 we can obtain

$$
\tilde{\psi}=B_{\theta} \frac{q_{s}^{\prime}}{q_{s}} \frac{W^{2}}{16}
$$

Since on the separatrix we have

$$
\psi_{x}=-\tilde{\psi} \quad \text { at } \zeta=0
$$

we obtain an equation between the $x$ and $\zeta$,

$$
x^{2}=\left(W^{2} / 8\right)(1-\cos \zeta) \quad \text { for } \psi=\psi_{x} .
$$

We first study the case when the ECE channel is crossing the island. Inside the island we assume the temperature is flat, the temperature fluctuation is simply given by

$$
\tilde{T}=-T^{\prime} x
$$

Using Eq. (18) and taking $\tilde{T}=0$ inside the island, we have

$$
\tilde{T}(\zeta)= \begin{cases}\mp T^{\prime} W \sqrt{\left[1-\cos \left(\zeta_{c}-\zeta\right)\right] / 8} & |\zeta|<\zeta_{c} \\ 0 & \zeta_{c} \leq \zeta \leq 2 \pi-\zeta_{c}\end{cases}
$$

Here, $-\operatorname{sign}$ is for $x>0$ and + for $x<0, \zeta_{c}$ is the crossing angle shown in Fig. 4. Therefore, the island width can be written as:

$$
W=\frac{\delta T}{\left|T^{\prime}\right|} \frac{\sqrt{8}}{\sqrt{1-\cos \zeta_{c}}}
$$


where $\delta T$ is the residual amplitude of temperature fluctuation as shown in Fig. 4(b). Now we study the case when island is located between two ECE channels. We noticed that for $x \geq W / 2$, the $T_{e}$ fluctuation follows Eq. (5), i.e.,

$$
\tilde{T}=-T^{\prime} \frac{\psi_{1} \cos \zeta}{B_{\theta}\left(1-q / q_{s}\right)}
$$

The $\psi_{1}$ [not necessarily equal to Eq. (16)] can be determined by matching $\tilde{T}$ to the inner fluctuation (19) at $x=W / 2(\zeta=\pi)$,

$$
\psi_{1}=B_{\theta} \frac{q_{s}^{\prime}}{q_{s}} \frac{W^{2}}{4}
$$

Substituting Eq. (23) into Eq. (22) we obtain

$$
W=\sqrt{2 d \frac{\tilde{T}}{\left|T^{\prime}\right|}} \quad \text { for } W \leq d .
$$

Here, the expansion $\left(1-q / q_{s}\right) \simeq-\left(q^{\prime} / q_{s}\right) d / 2$ has been used, $d$ is the distance of the two adjacent ECE channels, assuming the island is in the middle of two channels; $\tilde{T}$ is the peak to peak temperature fluctuation $\left(\tilde{T}=2 \sqrt{2} \tilde{T}_{R M S}\right)$. If the island is not in the middle $\tilde{T}$ is not equal in two channels and an averaged $\tilde{T}$ should be used. At the island separatrix $(d=W)$, Eq. (24) equals to Eq. (21) $\left(\zeta_{c}=\pi\right)$ as we expected. Due to the slab-like geometry assumed, both Eqs. (21) and (24) may fail for very large island case where the cylindrical effects are not negligible (e.g., asymmetric $\tilde{\psi}$ across the mode rational surface). 


\section{References}

[1] H. P. Furth, J. Killeen, and M. N. Rosenbluth, Phys. Fluids 6, 459 (1963).

[2] R. B. White, Rev. Mod. Phys. 58, 183 (1986).

[3] Z. Chang, E. D. Fredrickson, J. D. Callen, K. M. McGuire, M. G. Bell, R. V. Budny, C. E. Bush, D. S. Darrow, A. C. Janos, L S. Johnson, H. Park, S. D. Scott, J. D. Strachan, E. J. Synakowski, G. Taylor, R. M. Wieland, M. C. Zarnstorff, S. J. Zweben and TFTR Group, Nucl. Fusion 34, 1309 (1994).

[4] P. H. Rutherford, Phys. Fluids 73, 1903 (1973).

[5] M. C. Zarnstorff, M. G. Bell, M. Bitter, R. J. Goldston, B. Grek, R. J. Hawryluk, K. Hill, D. Johnson, D. McCune, H. Park, A. Ramsey, G. Taylor, and R. Wieland, Phys. Rev. Lett. 60, 1306 (1988).

[6] W. X. Qu and J. D. Callen, University of Wisconsin Plasma Report No. UWPR 85-5, 1985. (See National Technical Information Service Document No. DE6008946). Copies may be ordered from the National Technical Information Service, Springfield, VA 22161.

[7] R. Carrera, R. D. Hazeltine, and M. Kotschenreuther, Phys. Fluids 29, 899 (1986).

[8] Z. Chang, J. D. Callen, E. D. Fredrickson, R. V. Budny, C. C. Hegna, K. M. McGuire, M. C. Zarnstorff, and TFTR group, Phys. Rev. Lett. 744663 $(1995)$.

[9] R. J. La Haye, J. D. Callen, M. S. Chu, S. Deshpande, T. A. Gianakon, C. C. Hegna, S. Jardin, L. L. Lao, J. Manickam, D. A. Monticello, A. Pletzer, A. H. Reiman, O. Sauter, E. J Strait, T. S. Taylor, A. D. Turnbull and H. R. Wilson, at 16th IAEA conf., Montreal (1996) IAEA-CN-64/AP1-21. 
[10] D. A. Gates, B. Lloyd, A. W. Morris, G. McArdle, M. O’Brien, M. Valovic, C. D. Warrick, H. R. Wilson and The COMPASS-D and ECRH Teams, at 16th IAEA Conf. Montreal (1996) IAEA-CN-64/AP1-17.

[11] E. D. Fredrickson, K. McGuire, A. Cavallo, B. Grek, K.-I. Hattori, D. Johnson and A. W. Morris, Rev. Sci. Instrum. 59, 1797 (1988).

[12] A. Cavallo, R. C. Cutler, and M P. McCarthy, Rev. Sci. Instrum. 59, 889 (1988).

[13] F. J. Stauffer, D. A. Boyd, R. C. Cutler, and M. P. McCarthy, Rev. Sci. Instrum. 56, 925 (1985).

[14] J. D. Strachan, M. Bitter, A. T. Ramsey, M. C. Zarnstorff, V. Arunsalam, M. G. Bell, N. L. Bretz, R. Budny, C. E. Bush, S. L. Davis, H. F. Dylla, P. C. Efthimion, R. J. Fonck, E. Fredrickson, H. P. Furth, R. J. Goldston, L. R. Grisham, B. Grek, R. J. Hawryluk, W. W. Heidbrink, H. Hendel, K. W. Hill. H. Hsuan, K. P. Jaehnig, D. L. Jassby, F. Jobes, D. W. Johnson, L. C. Levinton, P. H. LaMarche, D. M. Manos, D. K. Mansfield, K. McGuire, D. H. McNeill, D. M. Meade, S. S. Medley, W. Morris, D. Mueller, E. B. Nieschmidt, D. K. Owens, H. Park, J. Schivell, G. Schilling, G. L. Schmidt, S. D. Scott, S. Sesnic, J. C. Sinnis, F. J. Stauffer, B. C. Stratton, G. D. Tait, G. Taylor,H. H. Towner, M. Ulrickson, S. von Goeler, R. Wieland, M. D. Williams, K. L. Wong, S. Yoshikawa, K. M. Young, and S. J. Zweben, Phys. Rev. Lett. 58, 1004 (1987).

[15] R. V. Budny, Nucl. Fusion 34, 1247 (1994) and references therein.

[16] H. P. Furth, P. H. Rutherford, and H. Selberg, Phys. Fluids 16, 1054 (1973).

[17] M. Zarnstorff, in Proceedings of Workshop on Local Transport Studies in Fusion Plasmas, Varenna, Italy, 30 August - 3 September 1993 (Societá Italina di Fisica, Bologna, Italy, 1993), p.257. 
[18] F. M. Levinton, Rev. Sci. Instrum. 63, 5157 (1992).

[19] H. R. Strauss, Phys. Fluids 24, 2004 (1981).

[20] C. C. Hegna and J. D. Callen, Phys. Plasmas 1, 2308 (1994).

[21] A. H. Glasser, J. M. Green, and J. L. Johnson, Phys. Fluids 19, 567 (1976).

[22] C. Ren, J. D. Callen, Z. Chang, E. D. Fredrickson, T. A. Gianakon, C. C. Hegna, K. M. McGuire, G. Taylor, and Zarnstorff, "Measuring $\Delta^{\prime}$ from $T_{e}$ Fluctuations in TFTR", to be published in Phys. of Plasmas. 


\section{List of Figures}

1 The basic MHD diagnostic systems in TFTR: 30 Mirnov coils (only shown the 15 poloidal array on one toroidal angle) and two 20channel ECE Grating Polychromator systems. The two ECE arrays are toroidally separated by $126^{\circ}$. Coil $L$ is used to calculate the magnetic island evolution. Also shown are the three plasma cross-sections in which TFTR routinely operated. . . . . . . . 21

2 A typical supershot plasma that developed an $m / n=3 / 2$ mode. (a) The tearing mode correlates with the performance degradation on $\beta_{N}=\beta_{t}(\%) a(m) B_{t}(T) / I_{p}(M A)$ and neutron rate $S_{n}$. (b) Evolution of the frequency spectrum from a magnetic coil. . . . . 22

3 (a) $T_{e}$ profile from ECE measurement and TRANSP $q$ profiles before and after a modification using Eq. (3). (b) $\tilde{T}_{e}$ fluctuations from the $18 \mathrm{ECE}$ channels. The MHD mode has a phase inversion between channel 12 and 13. It is an odd mode from the phase difference of channel 1 and 11. Shown also is the external magnetic measurement of the $3 / 2$ mode. . . . . . . . . .

4 (a) Island helical flux geometry. (b) $T_{e}$ fluctuations from three locations shown in (a). Here, $f$ is the mode frequency. The island width can be calculated from either the central channel $b$ that has a flat portion or from channels $a$ and $c$ using Eqs. (4) and (6)

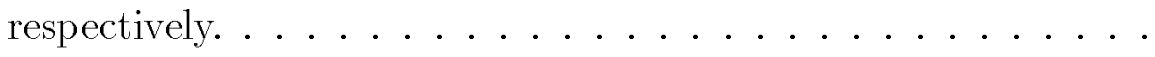

5 (a) Multi-channel ECE $\tilde{T}_{e}$ data at difference island evolution phases.

(b) Comparison of the island evolution from magnetic calculation and the outside-island ECE measurements $[$ Eq. (6)].......

6 Similar magnetic and ECE analyses as Fig. 5(b) for an $m / n=4 / 3$ mode (a) and $m / n=5 / 4$ mode $(b) \ldots \ldots 26$ 
7 (a) A plasma "Jog" experiment during the NBI phase. A $m / n=$ $2 / 1$ mode exists through the Jog phase. (b) "Continuous" $T_{e}$ and $\tilde{T}_{e}$ profiles can be obtained after a $t \rightarrow R$ mapping. Island width can be directly measured from $\tilde{T}_{e}(R)$. (c) The wave form data from three adjacent channels across the island during the jog, from which the island width can be calculated from Eqs. (4) and (6). . 27

8 (a) Comparison of $\Delta^{\prime}$ from a numerically calculation and analytic formula Eq. (8). The $q$ profile is shown in (b). This discharge developed a dominant $m / n=4 / 3$ mode in addition to the $1 / 1$ fishbone. . . . . . . . . . . . . . . . 28

9 Island width dependence of the $\Delta^{\prime}$ from numerical calculation (solid curves) and the Hegna-Callen analytic formula Eq. (12) (dashed curves). For the $2 / 1$ mode, the analytic curve is normalized to the numerical one at $w=0 \ldots \ldots \ldots \ldots$

10 Tearing mode $\tilde{T}_{e} / T_{e}$ eigenfunction for $m / n=3 / 2$ island from the mapped jog data. Also shown is the analytic fit to the exterior $\left(\left|r-r_{s}\right|>W / 2\right)$ region. $\ldots \ldots \ldots \ldots \ldots$

11 (a) Radial dependence of the four terms in Eq. (14). The sum of these four terms gives the $\Delta^{\prime}(W)(3 / 2$ mode $)$ in (b) at $2\left(r-r_{s}\right)=$ $W \simeq 5.2 \mathrm{~cm}$. Also shown is the calculated $\Delta^{\prime}(W) \ldots \ldots \ldots 31$

12 Similar jog data analysis as Figs. 11 and 12 for a $4 / 3$ island. The good fit (solid curves) gives negative $r_{s} \Delta^{\prime}$, which is in good agreement with the numerical calculation. As a comparison, a positive $\Delta^{\prime}$ would correspond to a bad fit (dashed curves) to the jog data.

13 Same analysis as Fig. 13 for a 2/1 mode. The obtained $\Delta^{\prime}$ from the jog data is more negative than the numerical result. Both zero width $\Delta^{\prime}(0)$ and finite width $\Delta^{\prime}(W)$ are shown. . . . . . . 


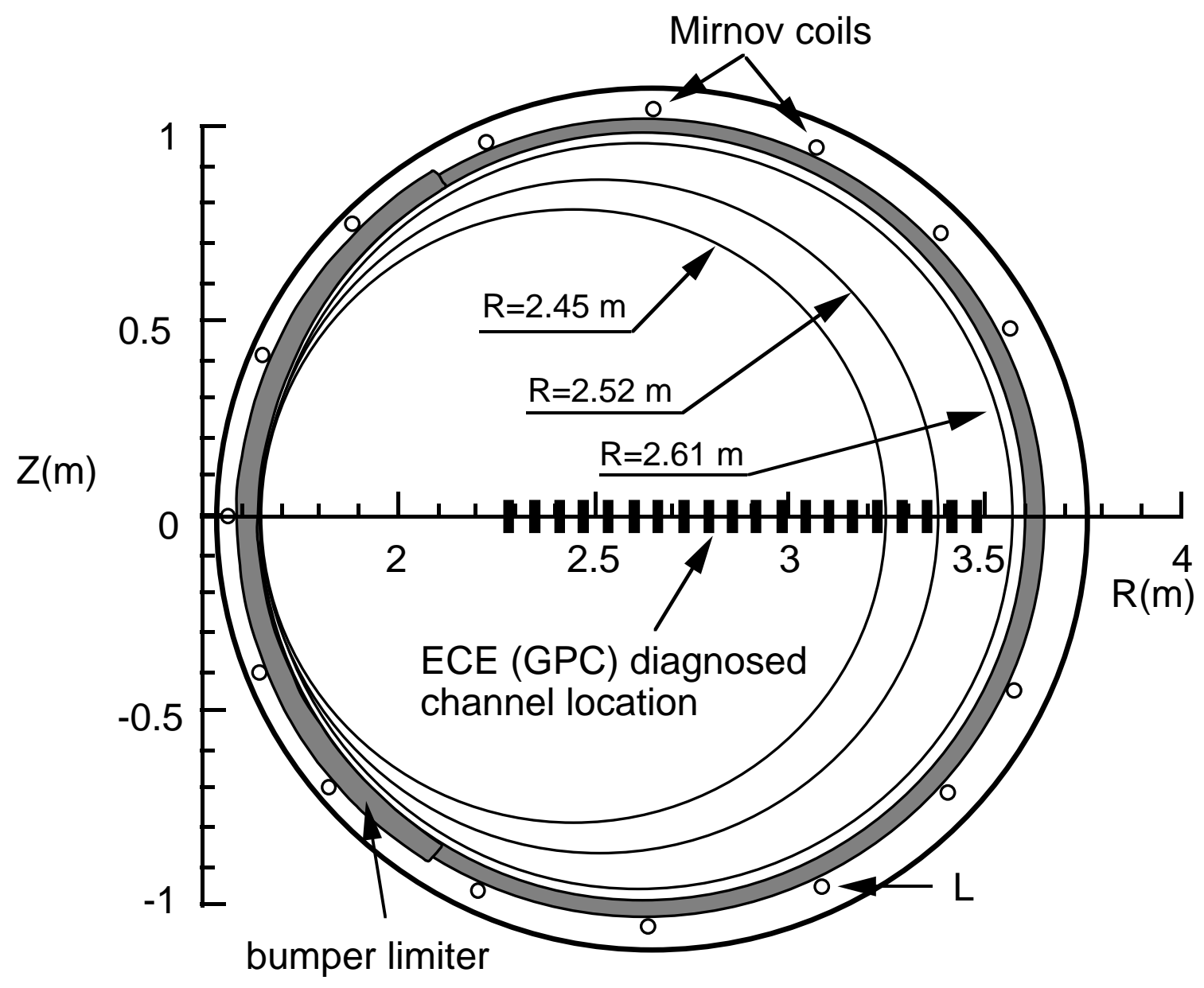

Z.Chang Fig.1 


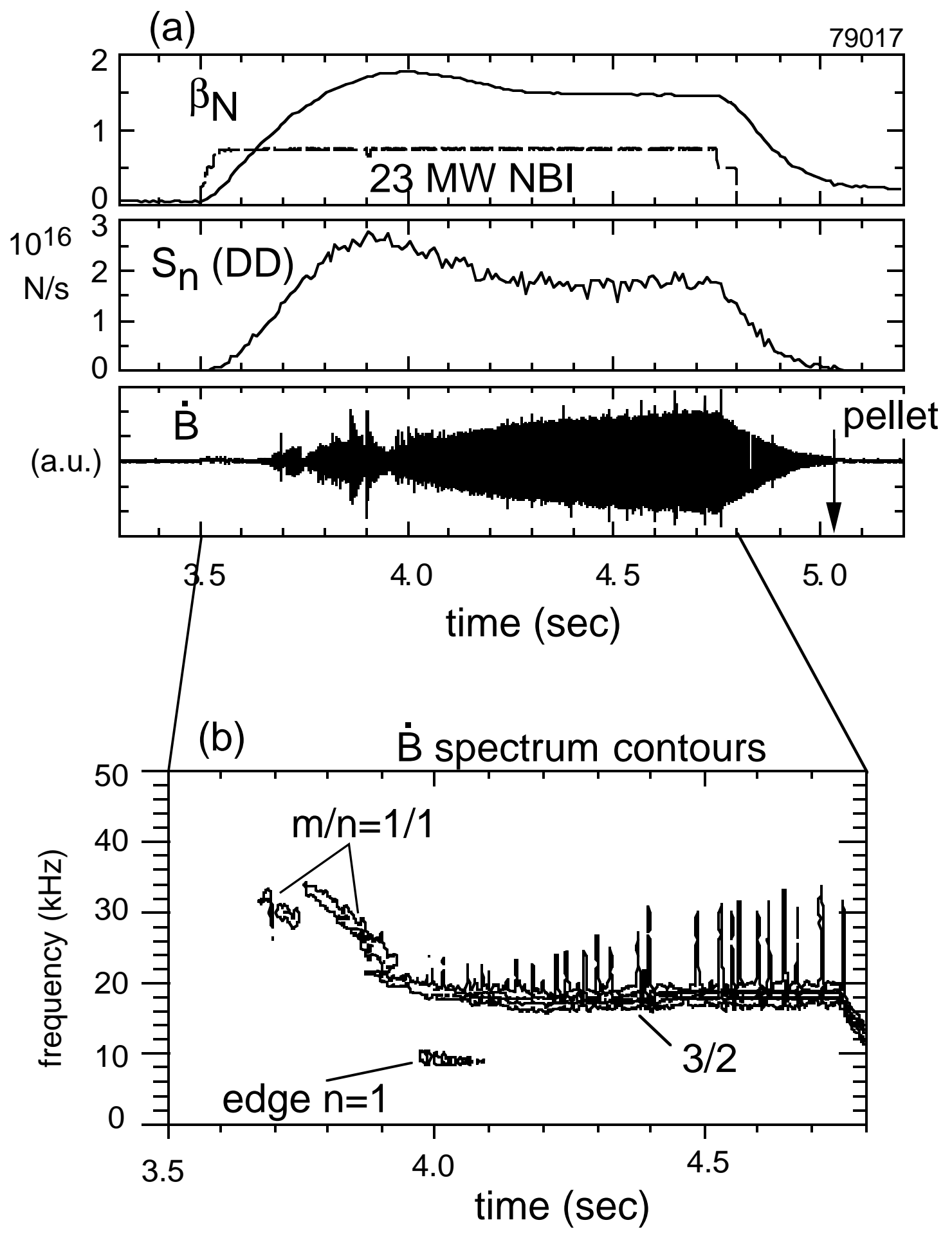

Z.Chang Fig. 2 

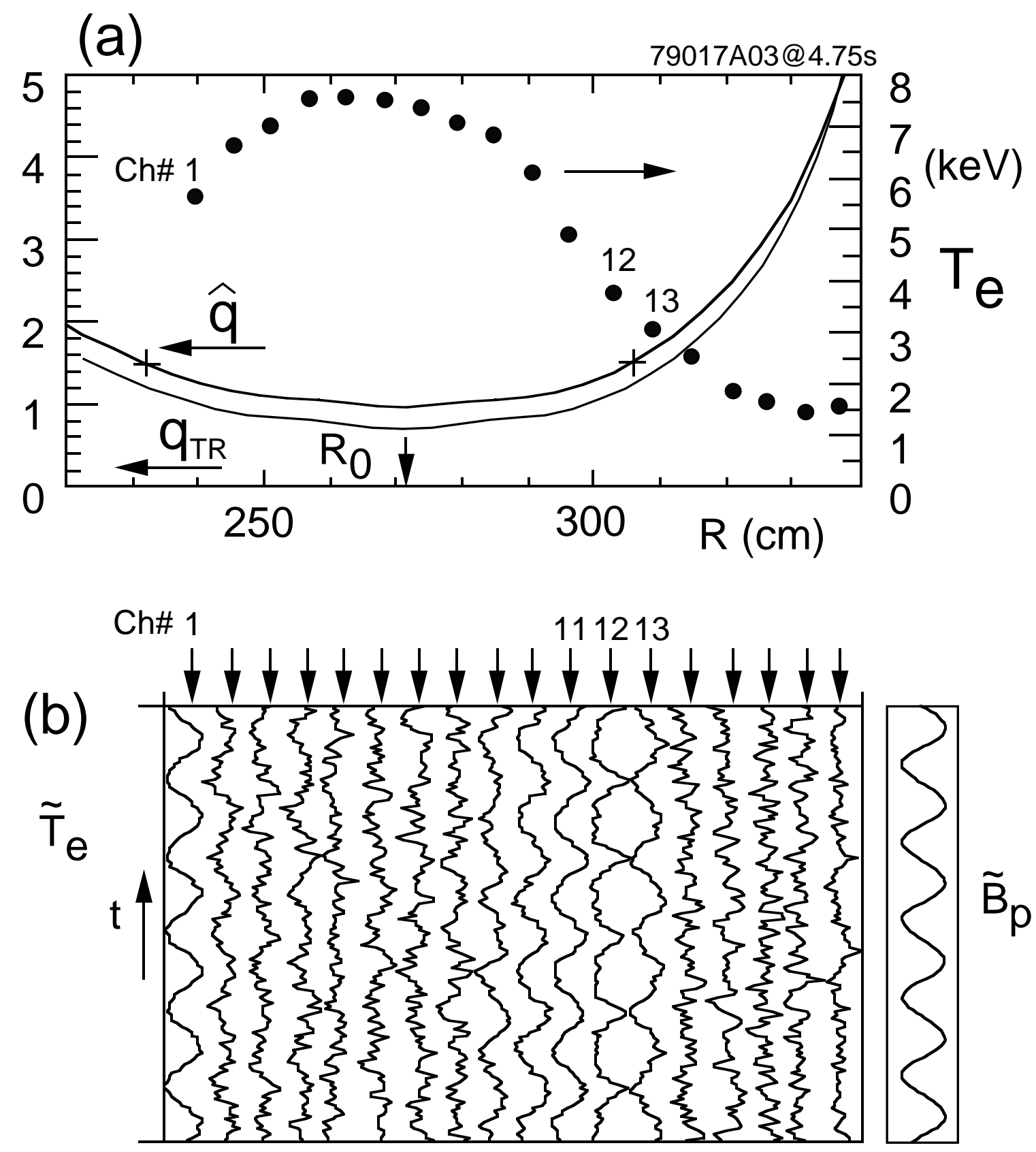

Z. Chang Fig.3 

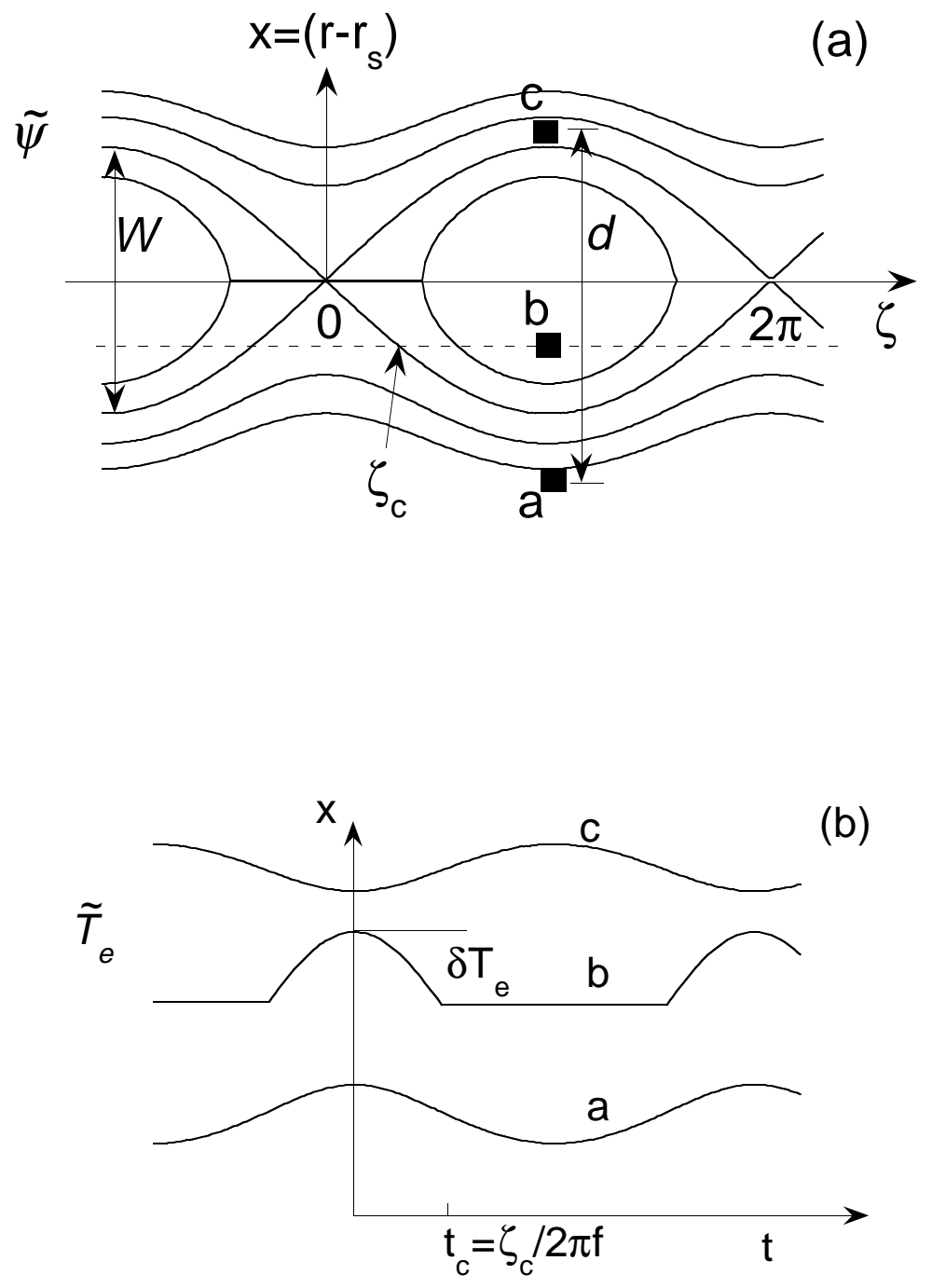

Z. Chang, Fig. 4 
(a)

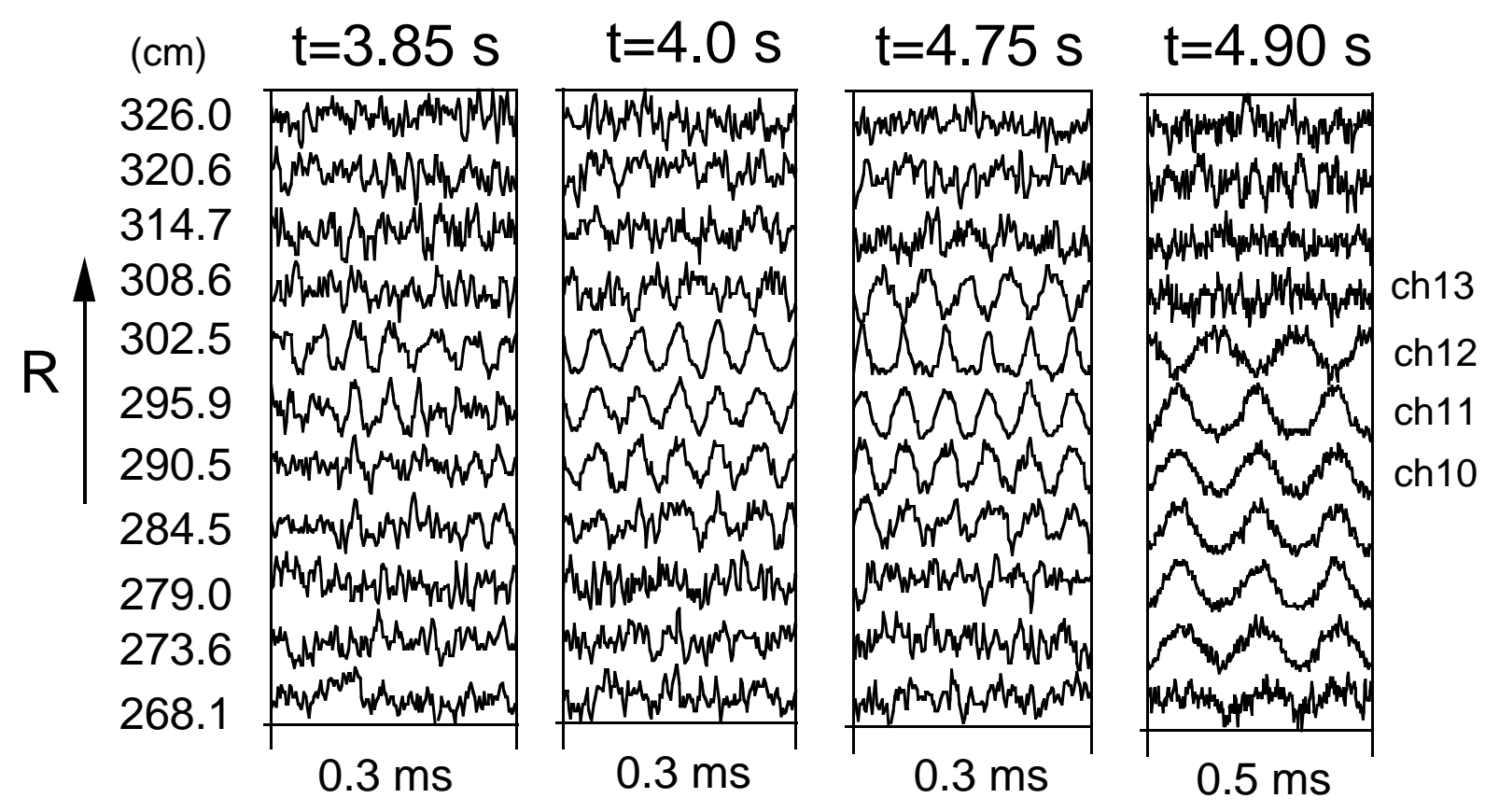

(b)

79017

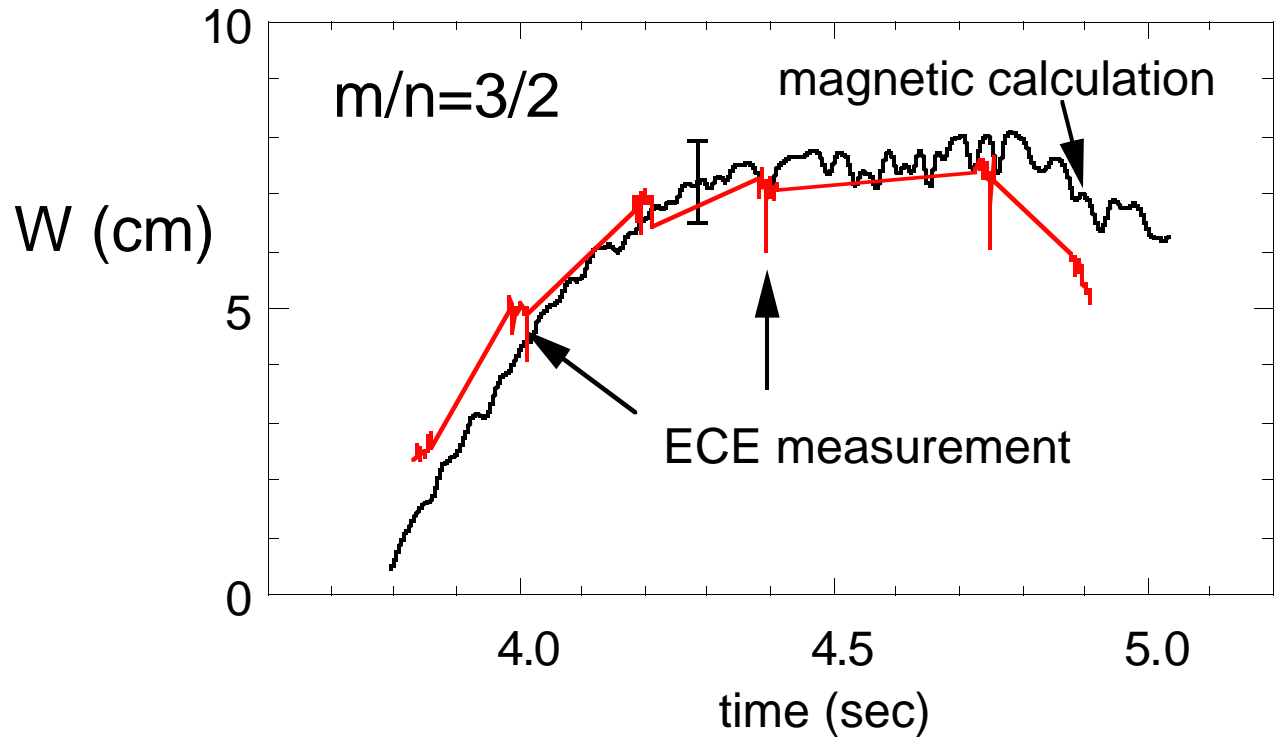

Z. Chang Fig.5 

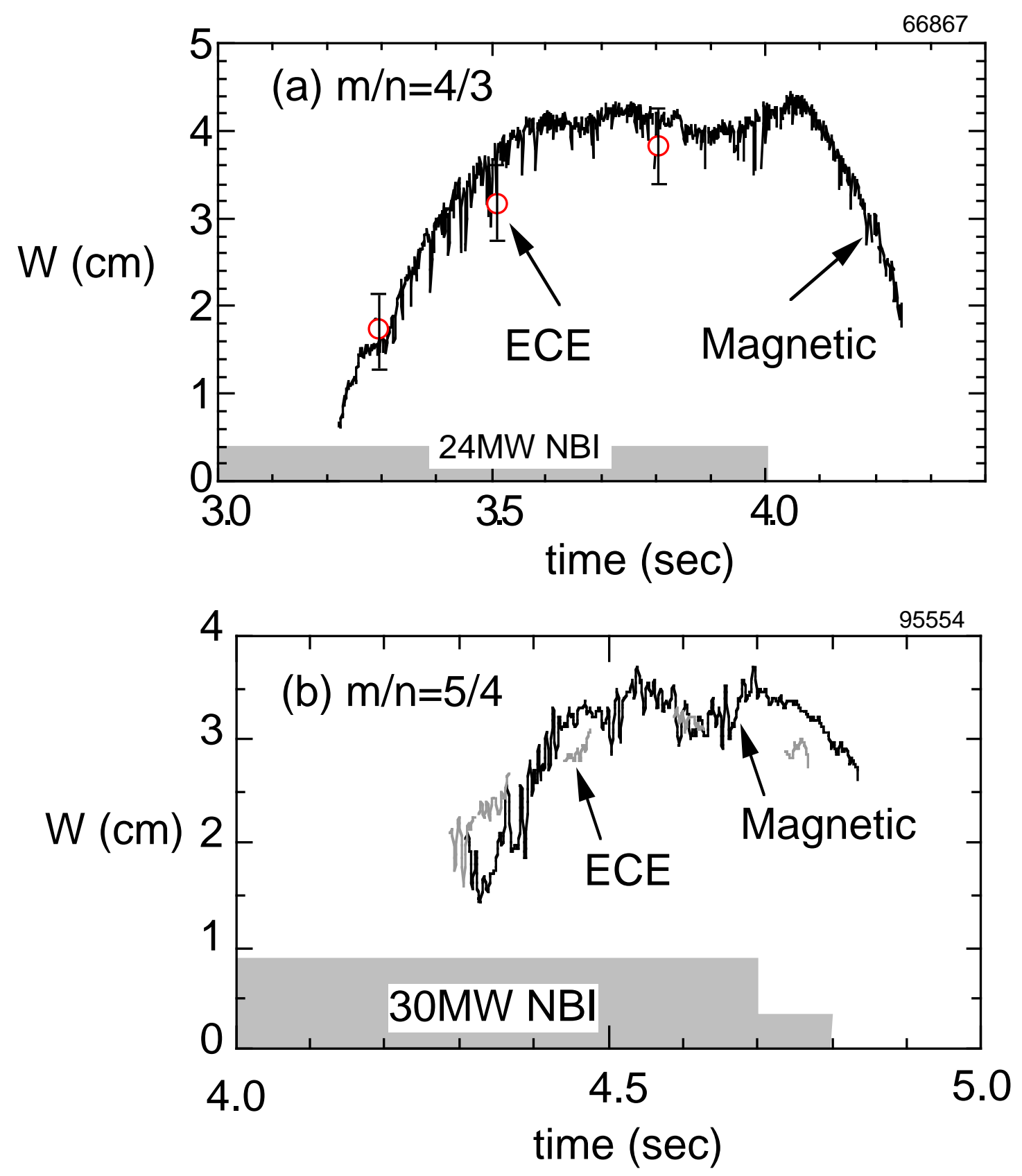

Z. Chang Fig. 6 
(a) 84665

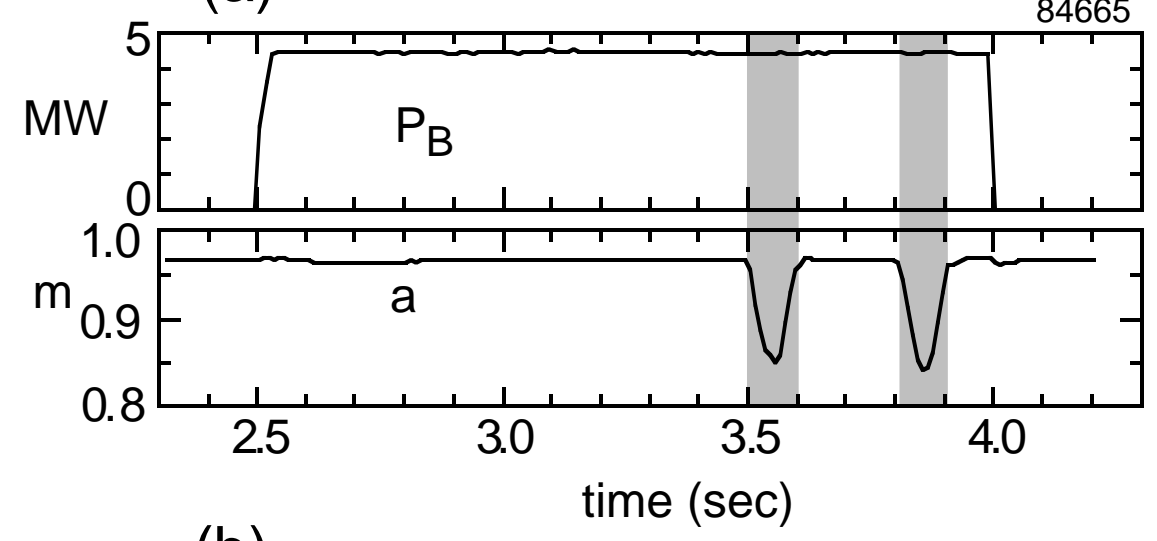

(b)
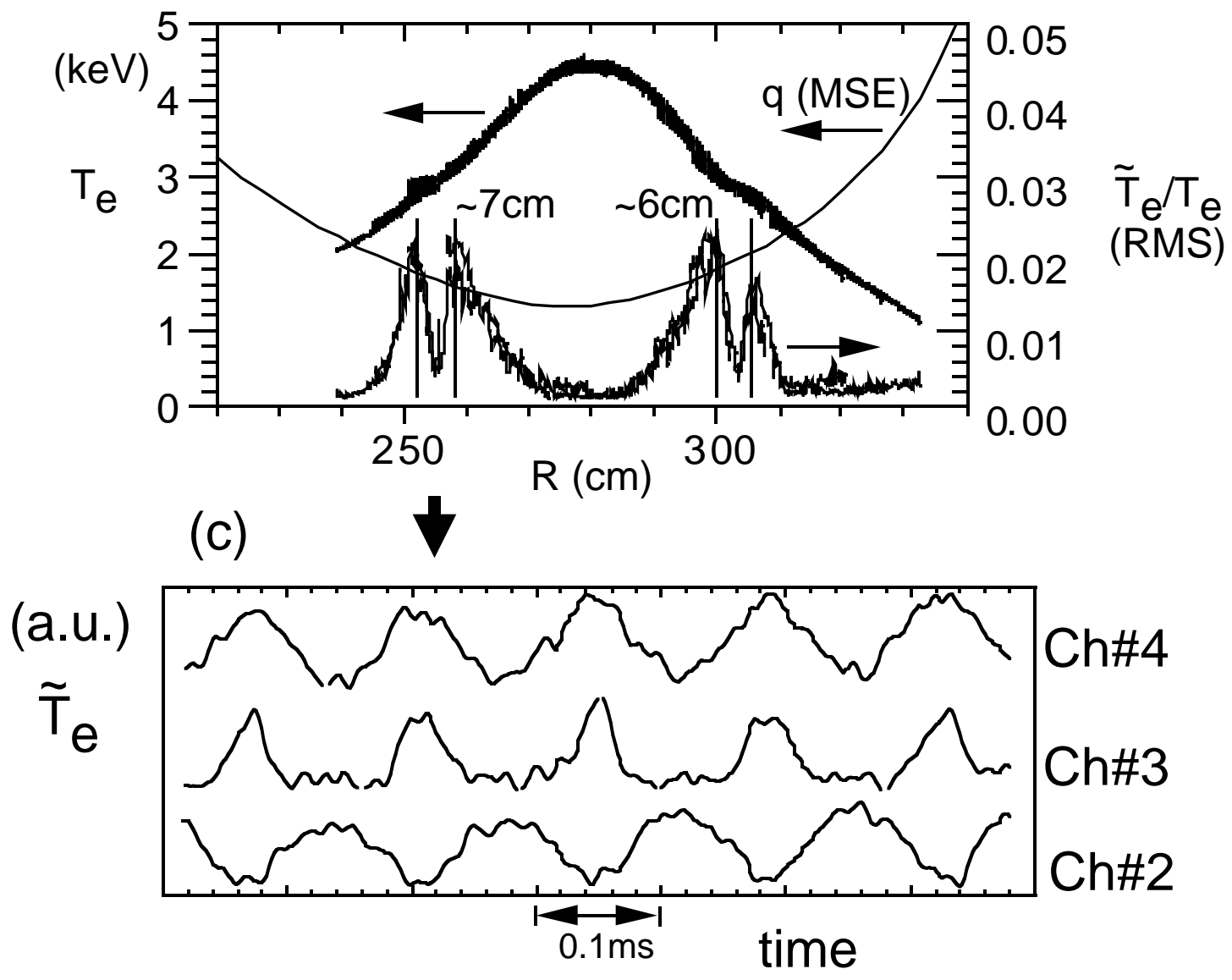

Z. Chang Fig. 7 
(a)

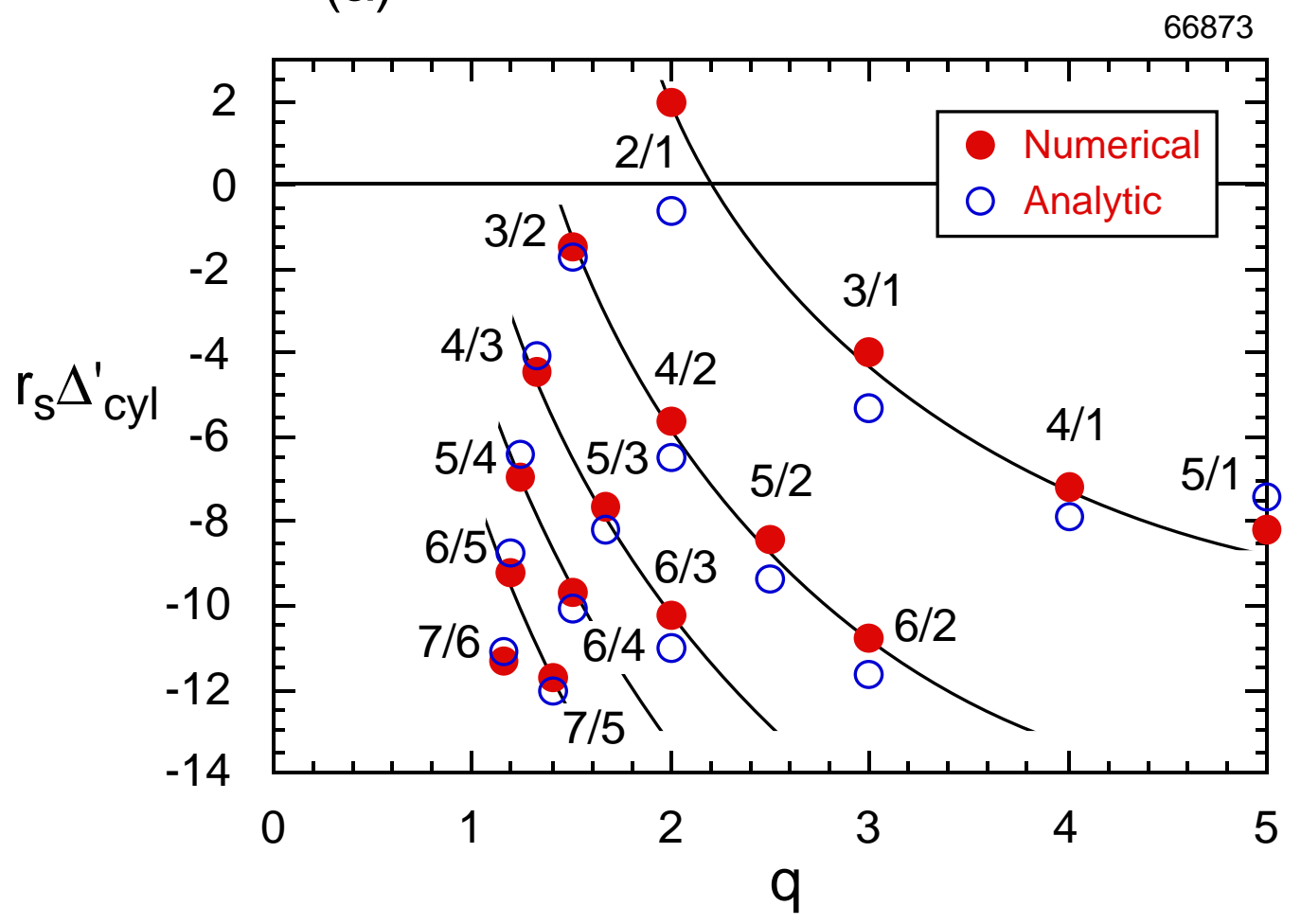

(b)

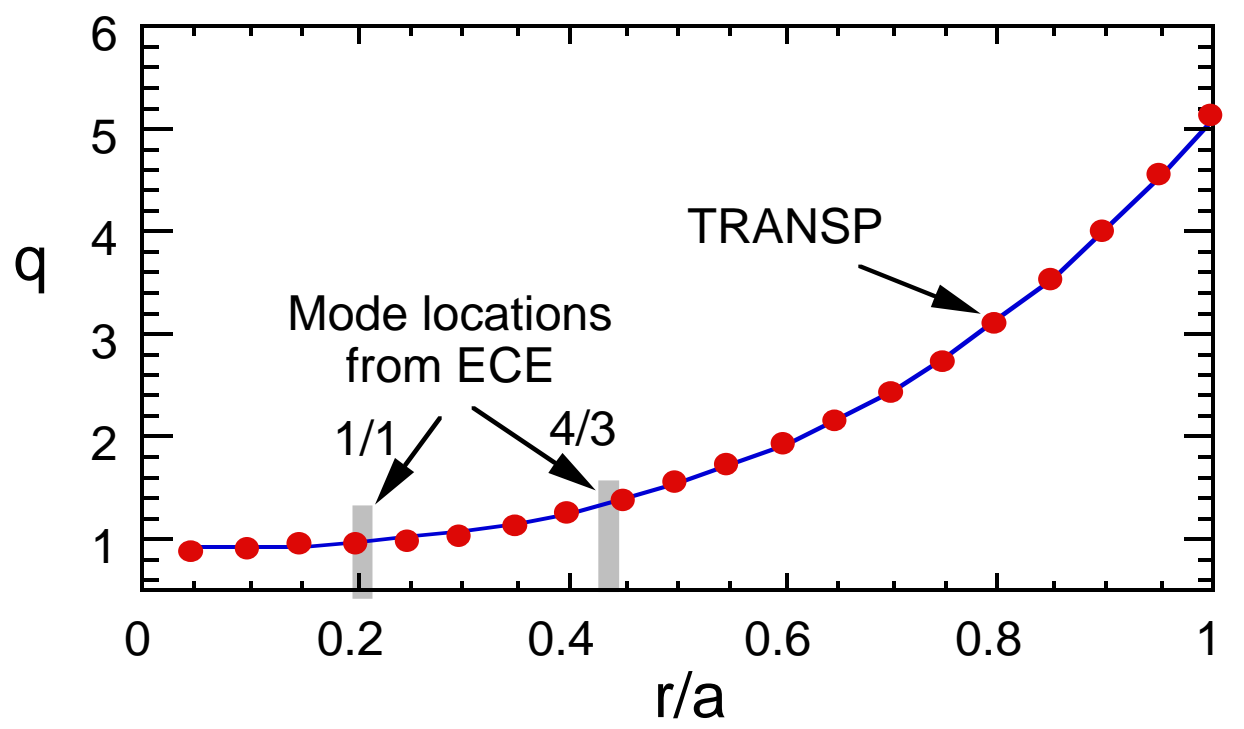

Z. Chang Fig. 8 


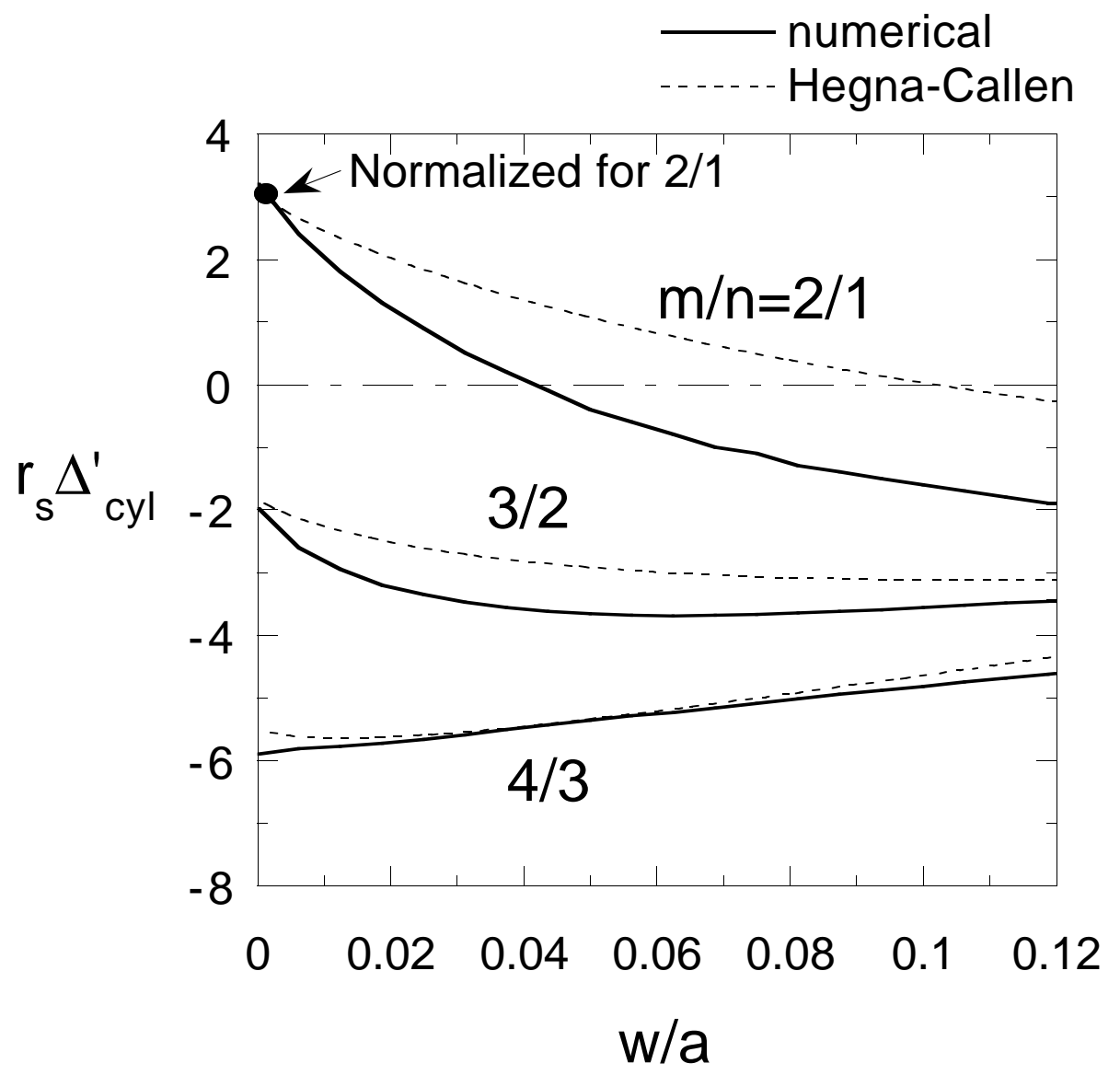

Z. Chang, Fig. 9 


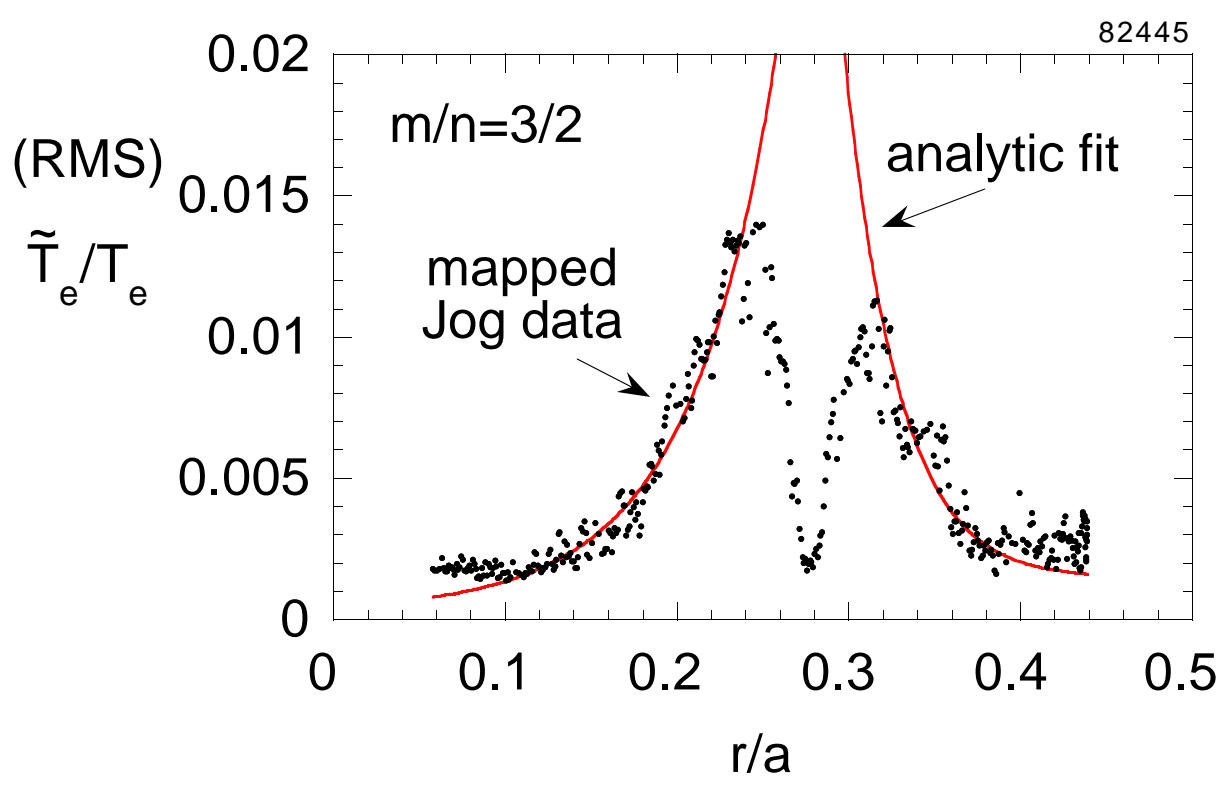

Z. Chang, Fig. $1 C$ 
(a)

82445

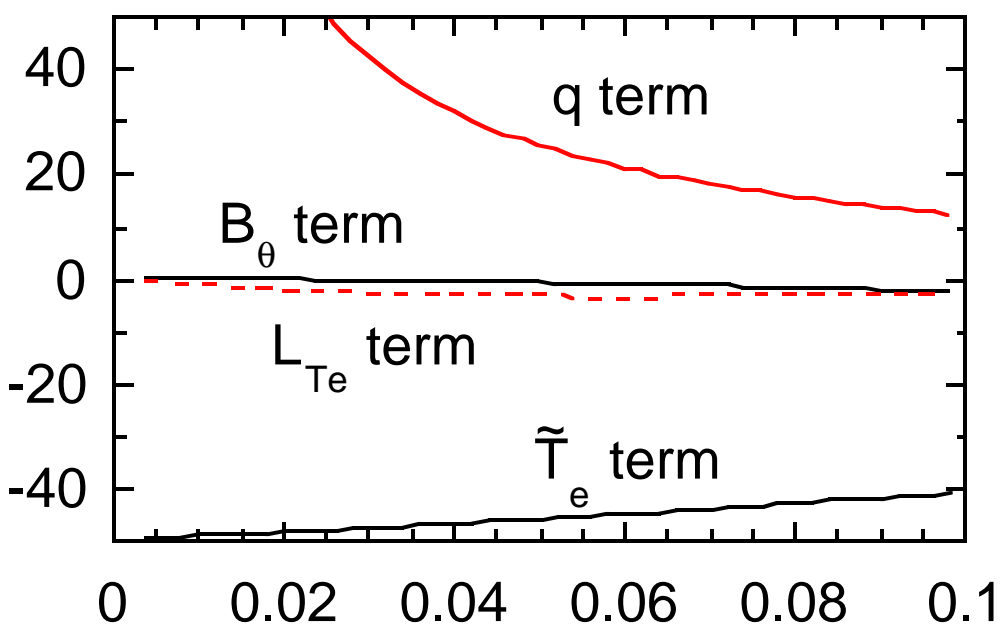

(b)

$$
x=\left(r-r_{s}\right) / a
$$

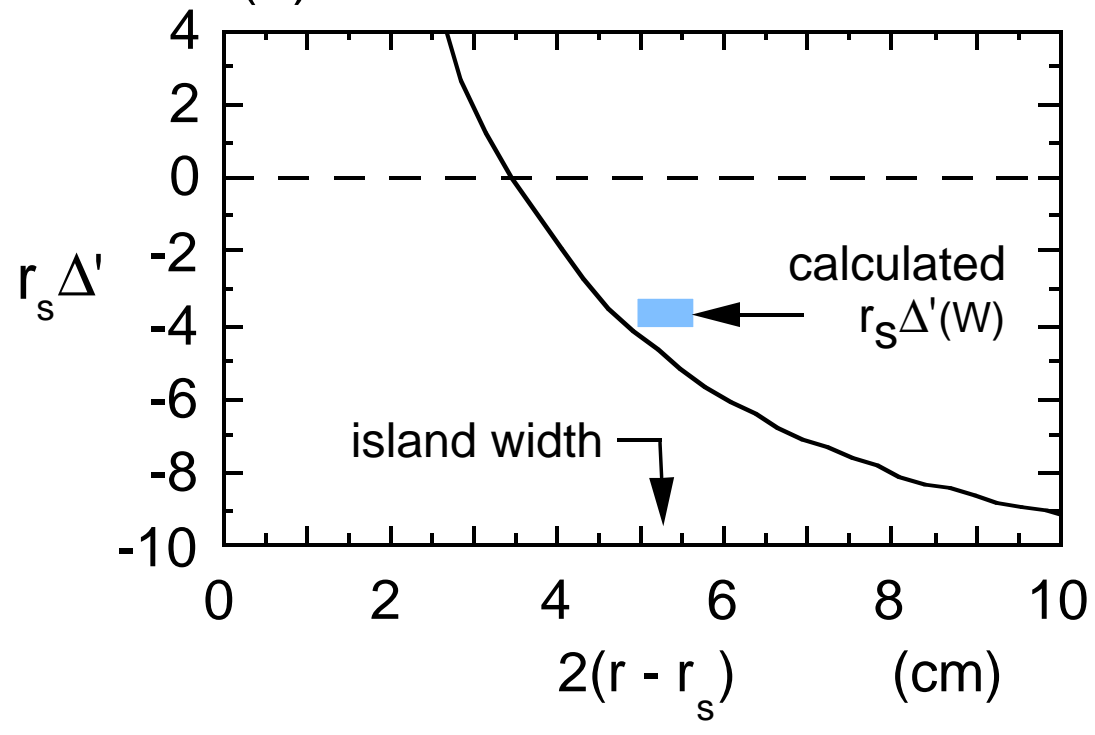

Z. Chang Fig. 11 
(a)

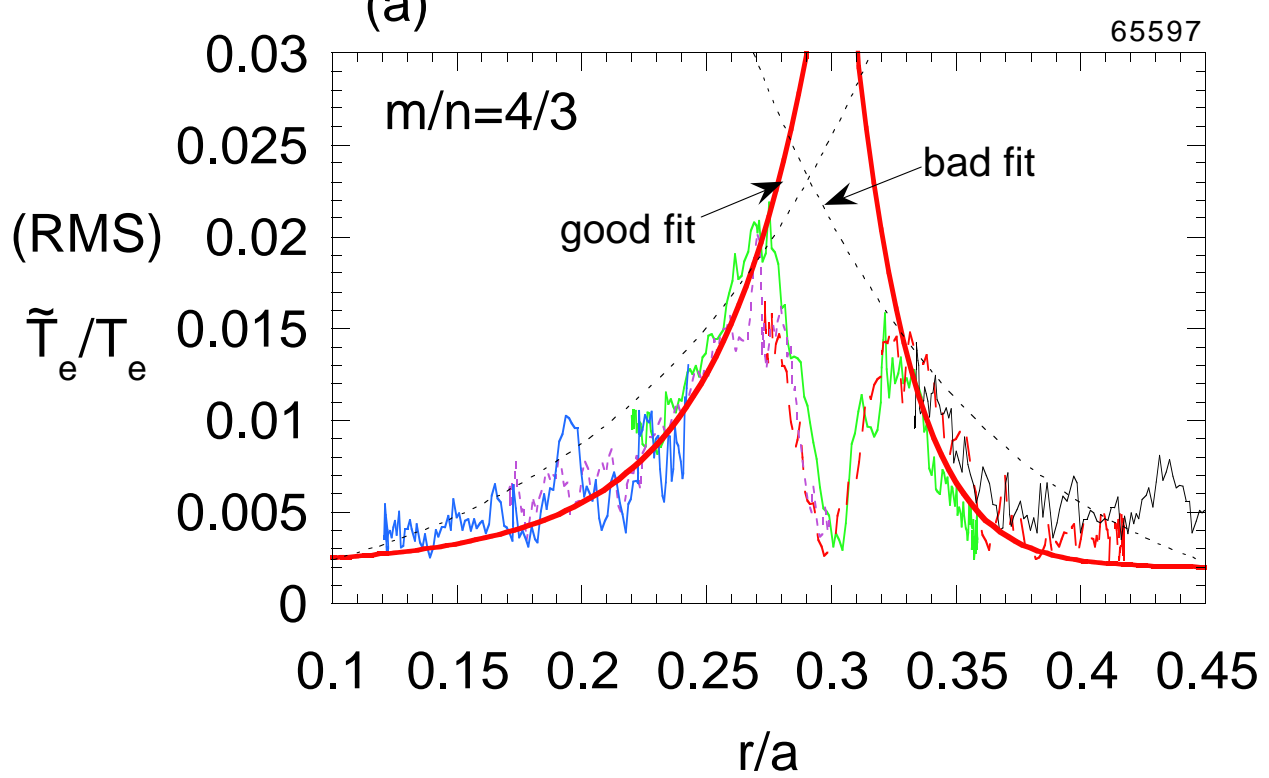

(b)

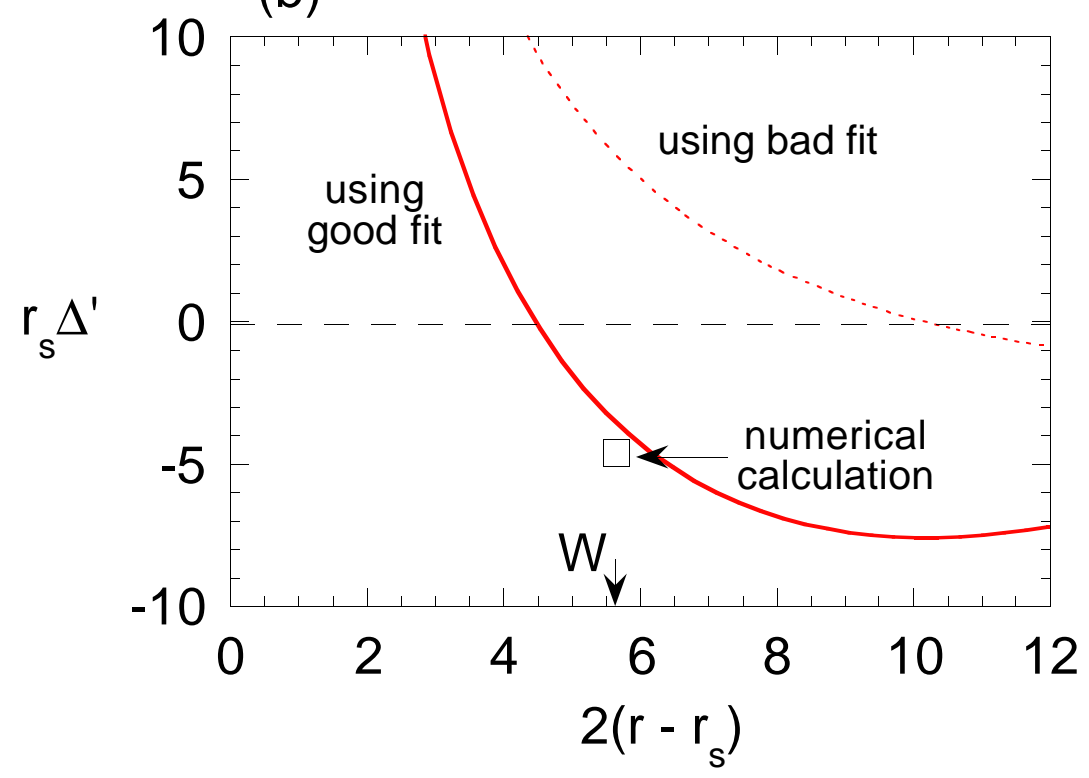

Z. Chang, Fig. $1 \epsilon$ 
(a)

84665

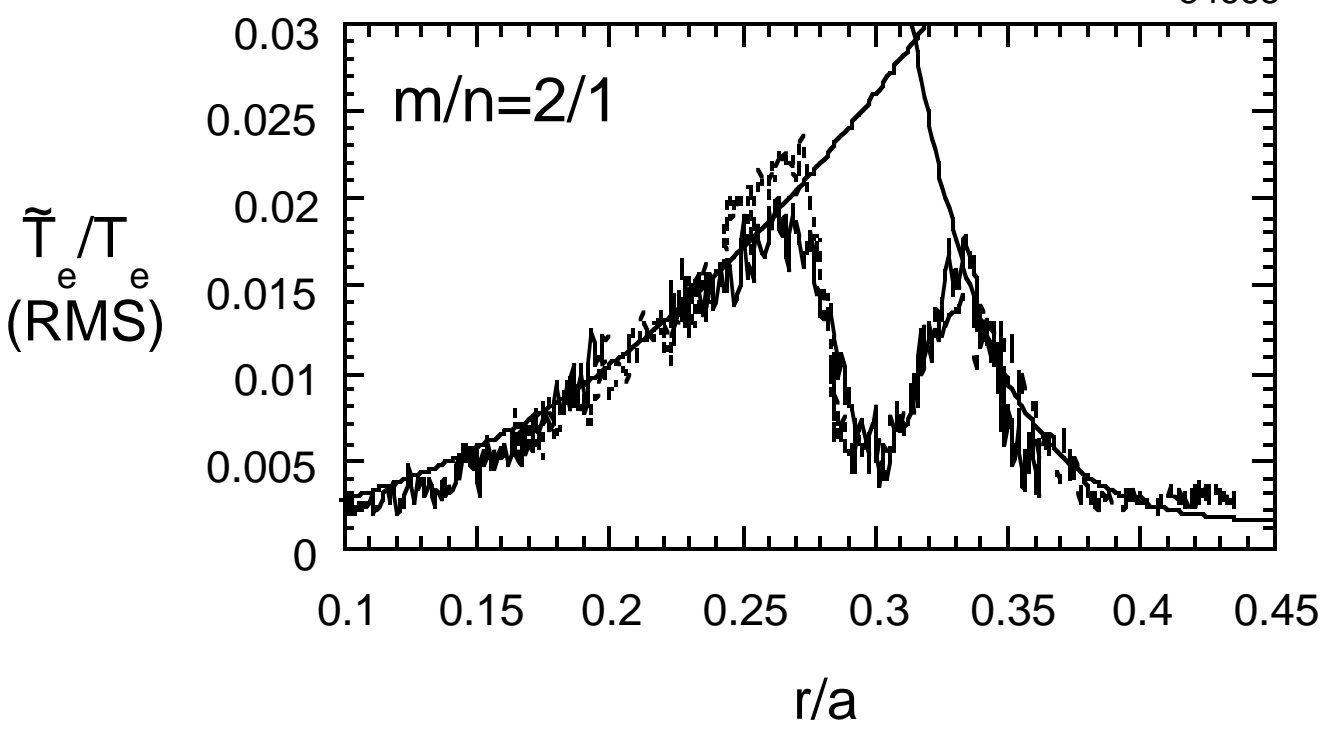

(b)

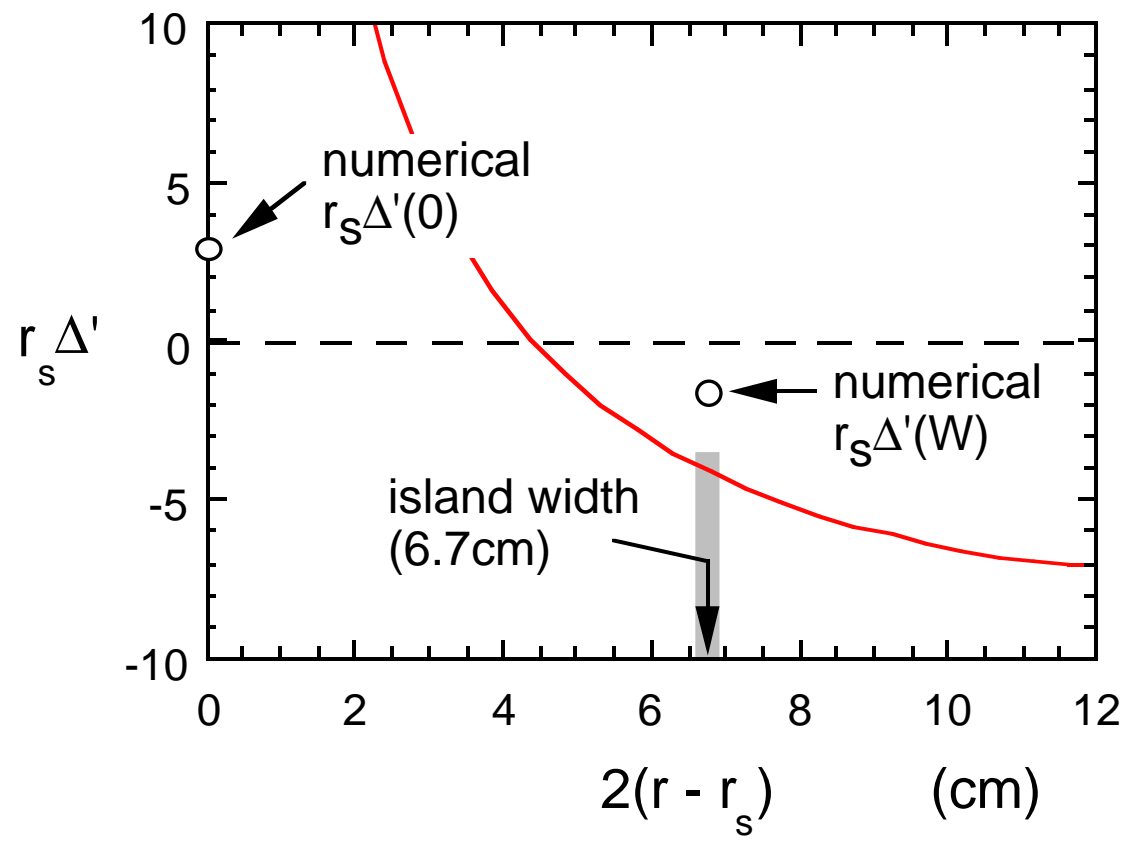

Z. Chang Fig. 13 